\title{
A Review of Disease Grading and Remote Diagnosis for Sight Threatening Eye Condition: Age Related Macular Degeneration Alauddin Bhuiyan*, Di Xiao and Kanagasingam Yogesan
}

Australian E-Health Research Centre, ICT Centre, Commonwealth Scientific and Industrial Research Organization, Underwood Avenue, Floreat 6014, Australia

\begin{abstract}
Age-related macular degeneration (AMD), a retinal disorder is the leading cause of vision loss in elderly people in developed countries. Currently, colour fundus imaging is used as a gold standard to assess individual for initial screening of AMD. In this paper, we examine the existing techniques for AMD screening using colour fundus imaging. The aim of the paper is to analyse and review the automated and semi-automated methods for detecting AMD pathologies such as drusen and geographic atrophy (GA) using colour fundus imaging. We intend to provide a brief description, highlighting the key points of the performance measurement and a framework for the existing research. We emphasize that to date; existing drug treatment can stop the progression of AMD and there is no drug or treatment to cure AMD. Therefore, the only feasible option is to prevent the incidence of AMD and avoid this fatal eye disease as well as unnecessary vision loss. For this, a mass screening system that enables remote diagnosis of AMD should be implemented for taking preventative measures in the early stage of AMD. Considering this, we discuss the open problems for automated AMD identification systems using colour fundus imaging and provided future directions for remote diagnosis and screening systems using colour fundus imaging and telemedicine platform.
\end{abstract}

\section{Introduction}

Age Related Macular Degeneration (AMD) is an eye disease characterized by the appearance of a progressive degenerative change in the macular area of the retina. This disease is the leading cause of legal blindness in people over 65 years in developed countries. It is difficult to know its exact prevalence because available studies may show different results as they have been done in different populations and in some cases, differences in diagnostic criteria and classification of the disease [1,2]. AMD affects 1 in 7 over the age of 50, with the incidence increasing with age [3]. The number is expected to increase by $\sim 1.5$ fold over ten years due to ageing population $[2,4]$. Whilst the use of supplements has been reported to slow progression in some groups of early stage of AMD, currently there are no specific interventions available to stop the development of AMD. The Age-Related Eye Disease Study (AREDS) has shown that specific anti-oxidant vitamin supplementation reduces the risk of progression from intermediate stages (defined as the presence of either many medium-sized drusen or one or more large drusen) to late AMD (defined as the presence of geographic atrophy - GA and/or choroidal new vascularisation $\mathrm{CNV}$ ) [5]. A brief definition of drusen, GA and CNV is provided in section 2.3.

As further strategies are developed that might make an impact on the risk of progression from early to late disease, it will be important that we are able to identify amongst the group of people with early signs, those that have a significant risk of progression. These people are the ones that should be targeted to enter treatment trials and use interventions as they become available. Currently, we do not have any such system which can mass screen population to measure early stage of AMD through regular eye check. A common situation is the cases are already late while the patients with AMD vision problem come to their ophthalmologists. Therefore, identifying people with early signs of the disease and then determining their risk based upon their fundus characteristics is important considering both the social and economic impact of AMD [6].

When determining the AMD stage as early or late, standard retinal images are graded for the presence of drusen (i.e., deposits of cellular waste that form beneath the retina [7].Drusen are further classified into subtypes by their appearance and then the total drusen area within the macular region is computed. Existing AMD grading systems require human grader's subjective manual interaction, which is time consuming and difficult to maintain intra or inter-rater agreement [8-11]. Due to the high degree of graders' involvement, the methods are prone to inaccuracy and poor repeatability if experienced graders are not used [12]. Therefore, an automatic system for detection and classification of drusen as well as AMD severity with less biased on grading error is a necessity. To allow large population based screening from urban and rural areas, we should incorporate telemedicine platform. A system that allows details of drusen type and size information can be used for monitoring drug effect on AMD treatment. Using vast collection of retinal images from population-based AMD studies, we should determine the sensitivity and specificity of retinal image features in identifying people at risk of late AMD.

The rest of the paper is organized as follows. In section 2, we provide a brief description of AMD definition and current diagnosis processes. Automated methods and existing grading protocols are surveyed in section 3. Section 4 provides a survey on existing telemedicine platforms related to AMD screening. Future research directions are provided in section 5. Finally, conclusions are drawn in section 6.

*Corresponding author: Alauddin Bhuiyan, Australian E-Health Research Centre, ICT Centre, Commonwealth Scientific and Industrial Research Organization, Underwood Avenue, Floreat 6014, Australia, Tel:+ 61893336149 ; E-mail: alauddin.bhuiyan@csiro.au

Received December 24, 2013; Accepted January 22, 2014; Published January 24, 2014

Citation: Bhuiyan A, Xiao D, Yogesan K (2014) A Review of Disease Grading and Remote Diagnosis for Sight Threatening Eye Condition: Age Related Macular Degeneration. J Comput Sci Syst Biol 7: 062-071. doi:10.4172/jcsb.1000139

Copyright: (c) 2014 Bhuiyan A, et al. This is an open-access article distributed under the terms of the Creative Commons Attribution License, which permits unrestricted use, distribution, and reproduction in any medium, provided the original author and source are credited. 


\section{AMD and Current Diagnosis Processes}

\section{AMD}

Age-related macular degeneration affects the macula i.e., the central part of the retina which is responsible for seeing final details and central vision (Figure 1). This disease is associated with aging and could gradually destroy sharp, central vision. The central vision is needed for seeing objects clearly and common daily tasks such as reading and driving.The AMD most frequently affects people over the age of 50 [1]. Figure 1 shows one example of the scenes viewed by normal and patients with AMD.

\section{Risk factors}

Therisk factorsfor AMD are age, gender (more women than men),family history of the disease, cigarette smoking,diet (low consumption of fruits and vegetables), excessive sunlight exposure, obesity, high blood pressure, cardio vascular disease and Alzheimer's disease [13].

\section{Types of AMD}

The stages of AMD can be defined based on the severity of AMD pathologies. The physiology of aging macula and the mechanisms implicated in the causes of AMD can be obtained from [14,15]. The clinical and histopathological features of AMD as well as its genetics and epidemiology can be found in Jager et al. [16]. Here, we mainly focus on the definition and pathologies of AMD for general readers.

In the early stages of macular degeneration, the transport of nutrients and wastes by the retinal pigment epithelium (RPE) slows down. As waste products accumulate under the retina, they form yellowish deposits called Drusen (Figure 2). Many people over the age of 60 will have some drusen. A portion of people with drusen may begin to experience mild vision loss [13]. Drusen can be defined as hard and soft drusen. Hard drusen are those which has sharp border (Figure 2-right). Soft drusen are with indistinct borders and larger size compared to hard drusen (Figure 3-left).

AMD Types: AMD occurs (at late stages) in two forms: 1) dry (atrophic or non-exudative) AMD and 2) wet (neovascular or exudative macular degeneration) AMD. Dry AMD accounts for $80-90 \%$ of all diagnosed patients, is characterized by sharply delineated areas of severe depigmentation or apparent absence of the retinal pigment epithelium (RPE) with a minimum diameter of $175 \mu \mathrm{m}$ [17].

Dry AMD: Dry AMD occurs when the light-sensitive cells in the macula become damaged and gradually blurring central vision of the affected eye. As the retinal pigment epithelium (RPE) continues to slow down in its transport of nutrients and wastes, the overlying photoreceptors become damaged. The size and number of drusen and geographic atrophies in the macula increase (Figure 3). Vision may be affected as RPE and photoreceptor cells are due to atrophy. As areas of retina lose function, patients begin to lose sight in certain areas in their central field of vision. Occasionally, a large region of cells is lost. This is called 'geographic atrophy' and it produces a blind spot in the central portion of vision (Figure 3-right). This blind spot is called scotoma.

Dry AMD generally affects both eyes, but vision can be affected in one eye while the other eye seems unaffected. $90 \%$ of the AMD people have dry AMD. Dry macular degeneration rarely causes severe vision impairment or blindness [13].

Wet AMD: It occurs when abnormal blood vessels behind the retina start to grow under the macula. The proliferation of these new blood vessels is called choroidal neovascularisation (CNV). These new blood vessels tend to be very fragile and often leak blood and fluid. The blood and fluid raise the macula from its normal place at the back of the eye, causing the retinal surface to become uneven. As a result, objects in that portion of the visual field may appear wavy or distorted. The neovascularisation may even break through some of the retinal layers. Blind spots may appear in vision if portions of the retina become damages by the CNV [13]. These features could be best diagnosed using high quality retinal photos of the macula. With advanced imaging technologies such as Spectral Domain Optical Coherence Topography (SD-OCT) can produce high sensitivity on extraction of these pathologies [18].

\section{Diagnostic tests for dry and wet AMD}

The below tests (current methods) are widely used in the eye clinics for AMD. In addition to these tests, OCT imaging and faceto-face evaluation of the fundus are performed in the clinics to make comprehensive examination for AMD.Besides these tests, a 3D computer-automated visual field test has been proposed [19].

Visual acuity (general vision loss): A decrease in best corrected

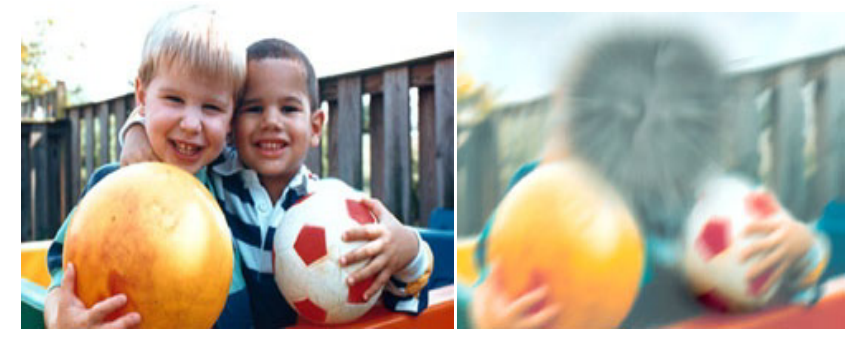

Figure 1: Normal vision (left) and scene viewed by person with AMD (right).
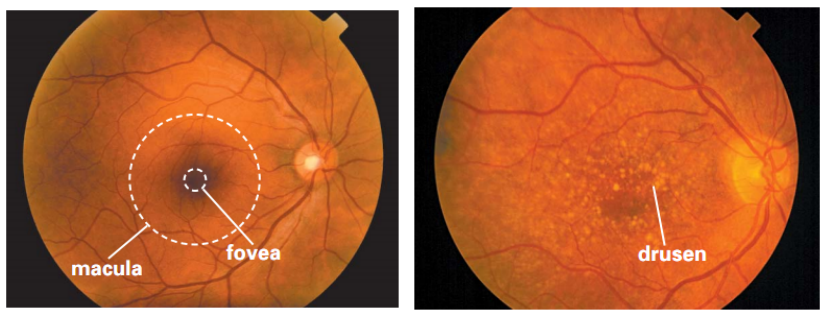

Figure 2: Photograph shows a normal healthy retina (left) and Image from an AMD patient with yellow drusen (right).
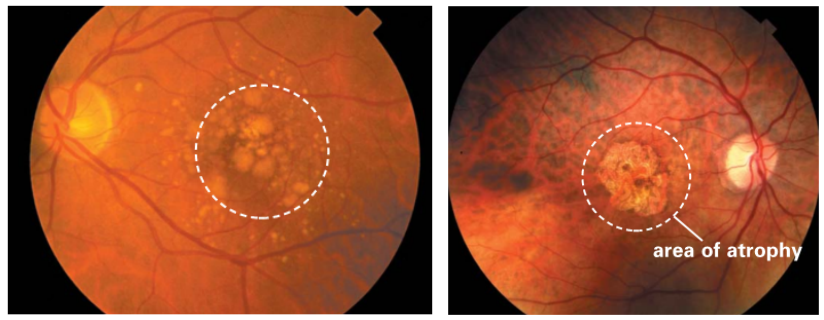

Figure 3: Retinal image showing many Drusen in the macula (left) and geographic atrophy (right). 
visual acuity (VA) score from baseline, of 15 or more letters (baseline equivalent $6 / 9$ or better, to $6 / 18$ or worse) [20]. The computer-aided Snellen chart has been already implemented (Figure 4).

Amsler grid test (wet AMD): The pattern of the grid resembles a checkerboard (Figure 5 - left). You will cover one eye and stare at a black dot in the centre of the grid. While staring at the dot, you may notice that the straight lines in the pattern appear wavy (Figure 5 right). Some lines may be missing. These may be signs of AMD.

Slit lamp: A slit lamp is a special microscope that enables the doctor to examine the different parts of the eye under magnification (Figure 6). When used with special lenses, the slit lamp gives the doctor a highly magnified view of the retina. Usually, dilating drops are administered and given time to work, the eye doctor seats the patient at the device [13].

Retinal colour photography or imaging: A fundus photo of the macula could be obtained using the low cost retinal camera in the market. This device is easy-to-operate by trained nurse or healthcare professionals. Figure 6-right shows a colour fundus image.

Other imaging modalities: Following the initial examinations, the ophthalmologist further examines the patient with advance imaging so that the screening should not miss any cases with AMD and its severity. The imaging modalities include:

Fluorescence Angiography [13]

Optical Coherence Tomography (OCT) [13]

Autofluorescence Imaging [21]

Thermal Infrared Imaging [22]

Doppler Imaging [23]

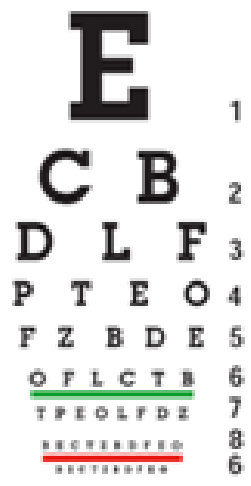

Figure 4: Snellen chart for testing Visual Acuity.

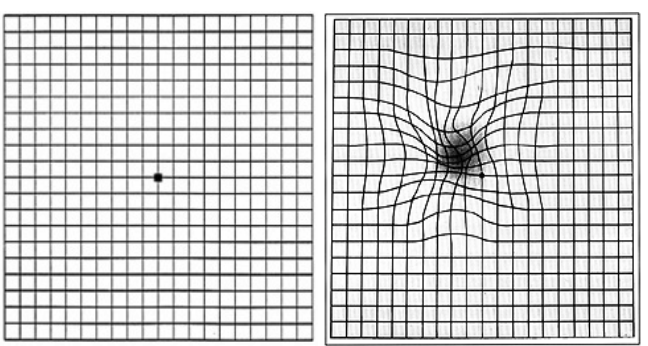

Figure 5: Amsler Grid: normal vision (left), AMD patient sees (right).

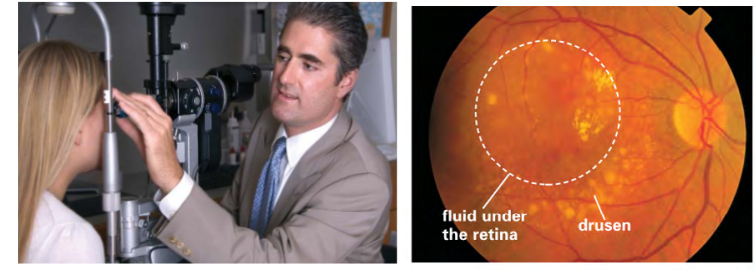

Figure 6: Slit lamp examination (left) and retinal colour photograph shows many drusen and fluid under the retina (right) (images taken from [13]).

A discussion on different imaging methodologies and their role on AMD screening and diagnosis can be obtained from [24]. Colour imaging is the gold standard and widely used for initial screening of AMD. In our discussion, we will focus on colour fundus image analysis algorithms for AMD pathology detection.

\section{AMD Grading Techniques}

Automated AMD grading techniques so far mainly focus on drusen detection using colour fundus images. A number of articles have been published on drusen detection using retinal colour fundus imaging. However, a few of them are utilized drusen size, area and actual number in the image to grade AMD severity. Overall, we can group the automated methods for drusen detection and quantification approaches. We note that a few of the techniques can classify soft drusen as distinct and indistinct. Thus, most of these automatic techniques are constrained in determining drusen segmentation level and drusen absence and presence in the retina.

In this section, we briefly introduce the international classification and grading system for age related maculopathy and age related macular degeneration. Following this, we discuss the existing techniques for drusen detection and identification.

\section{Protocols for colour retinal image grading for AMD}

The international classification and grading system divides the macula area within three circles which are centred on the fovea, of diameters corresponding to $1,000 \mu \mathrm{m}, 3,000 \mu \mathrm{m}$ and $6,000 \mu \mathrm{m}$ on the retina [25]. Lesions are graded within each of the central, inner or outer circles. The predominant drusen type is classed as the most common type of drusen present within the outer circle. The drusen are classified as hard drusen with size $<63 \mu \mathrm{m}$, intermediate soft drusen $63-125 \mu \mathrm{m}$, large semi-solid drusen $125-250 \mu \mathrm{m}, 250-500 \mu \mathrm{m}$ and $>500 \mu \mathrm{m}$. Other pathologies such as hyperpigmentation, GA and neovascular AMD is graded as presence or absence in the retinal image.

AREDS study defines the AMD category as 1 to 4 - normal to severe AMD based on the drusen, GA, RPE and neovascularization. The study defines AMD category 1 if there is no drusen exists or drusen size < $63 \mu \mathrm{m}$ and total area covered by drusen is $<125 \mu \mathrm{m}$. AMD category 2 or intermediate is defined as drusen size $>=63 \mu \mathrm{m}$ and $\langle 125 \mu \mathrm{m}$ and drusen area $>=125 \mu \mathrm{m}$ but GA absent. AMD category $3 \mathrm{a}$ is defined as intermediate $>=63 \mu \mathrm{m}$ and $<125 \mu \mathrm{m}$, drusen area $360 \mu \mathrm{m}$ diameter circle if soft distinct drusen are present and $>=656 \mu \mathrm{m}$ diameter circle if soft indistinct drusen are present. And other categories are defined as in Table 1.

\section{Drusen detection techniques}

Existing algorithms for AMD detection use colour imaging mainly for drusen detection and grading. These automated and 


\begin{tabular}{|c|c|c|c|c|}
\hline \multirow{2}{*}{$\begin{array}{l}\text { AMD } \\
\text { Category }\end{array}$} & \multicolumn{2}{|l|}{ First Eye } & \multirow{2}{*}{ Pigment Abnormalities } & \multirow[b]{2}{*}{ Second Eye } \\
\hline & Drusen Size & Drusen Area & & \\
\hline 1 & $\begin{array}{l}\text { None or small } \\
(<63 \mu \mathrm{m})\end{array}$ & $\begin{array}{l}<125 \mu \mathrm{m} \text { diameter circle }(\approx 5-15 \text { small } \\
\text { drusen })\end{array}$ & none & Same as first \\
\hline 2 & $\begin{array}{l}\text { small }(<63 \mu \mathrm{m}) \\
\text { or } \\
\text { Intermediate }(\geq 63,<125 \mu \mathrm{m}) \\
\text { None required if pigment } \\
\text { abnormalities present }\end{array}$ & $\begin{array}{l}\geq 125 \mu \mathrm{m} \text { diameter circle } \\
\text { At least one druse }\end{array}$ & $\begin{array}{l}\text { Absent or present but GA } \\
\text { absent }\end{array}$ & Same as first or category 1 \\
\hline $3 a$ & $\begin{array}{l}\text { Intermediate }(\geq 63,<125 \mu \mathrm{m}) \\
\text { Or } \\
\text { Large }>125 \\
\text { None required if non central GA } \\
\text { present }\end{array}$ & $\begin{array}{l}\geq 360 \mu \mathrm{m} \text { diameter circle if soft } \\
\text { indistinct drusen are present } \\
(\approx 20 \text { intermediate drusen }) \\
\geq 656 \mu \mathrm{m} \text { diameter circle if soft } \\
\text { indistinct drusen are absent. }\end{array}$ & $\begin{array}{l}\text { Absent or present but GA } \\
\text { absent }\end{array}$ & Same as first or category 1 or 2 \\
\hline $3 b$ & First eye same category as $3 a$ & & & $\begin{array}{l}\text { Visual Acuity }<20.32 \text { due to AMD; or unicolour } \\
\text { disqualifying disorder present }\end{array}$ \\
\hline $4 a$ & $\begin{array}{l}\text { First eye same category as } 1,2 \text {, } \\
\text { or } 3 a\end{array}$ & & & Advanced AMD \\
\hline $4 b$ & $\begin{array}{l}\text { First eye same category as } 1,2 \text {, } \\
\text { or } 3 a\end{array}$ & & & $\begin{array}{l}V A<20 / 32 \text { due to } A M D \text {, but advanced } A M D \text { not } \\
\text { present }\end{array}$ \\
\hline
\end{tabular}

Table 1: AREDS AMD categories.

semi-automated drusen detection and grading using colour fundus imaging involve image normalization and enhancement, macula area mapping, drusen segmentation and drusen area computation. Image normalization is an essential step as a bright source in fundus camera may cause shading in fundus imaging because of the irregularity of retinal shape. Contrast enhancement is another essential step to improve the appearance of details in retinal image before processing. Macula area can be automatically located and its regions can be mapped by using the detected optic disc information and major blood vessel traversal path. Drusen detection is applied using colour and texture feature extraction and feature vector clustering or machine learning based classification. Drusen are also segmented by thresholding and edge detection algorithms.

A number of methods [26-33] which mainly identifies images as absence or presence of drusen. Drusen area were analyzed and drusen are classified as large drusen, medium and small drusen in [29,34]. A number of methods have been proposed in [35-40] which are focused on drusen pixel level classification to segment regions in the image as drusen and non drusen, and analyze the accuracy as sensitivity and specificity of the method with hand labelled ground truth images.Our study only found two publications - one for automatic detection of dry and wet AMD [41] and one for soft and hard drusen classification [29] which are very important information for automated measurement of AMD severity. In addition, these studies were not validated against large dataset.

In the following discussion, we have provided a review on automated algorithms for drusen detection using colour retinal images. We categorize the methods based on the main image processing steps such as texture, edge, mathematical morphology, intensity thresholding and clustering based segmentation techniques.

\section{Texture based segmentation}

Texture generally describes second order property of surfaces and scenes, measured over image intensities [42]. Considering the difference of texture between the drusen and background researchers have applied a bank of Gabor filters, wavelet feature extraction, amplitude modulation - frequency modulation to extract texture features and applied suitable classification of these features.

Brandon and Hoover [26] developed a multi-level method to detect drusen applying wavelet-based feature extraction and classification. Mexican hat wavelet is applied to obtain four One Dimensional (1D) responses centred on the pixel for a given dilation: one horizontal, one vertical and two diagonals. A feature vector containing a set of values from the product of the four 1D responses by a set of given discrete dilations is computed and defined as the pixel's "wavelet signature", which is fed into a Feed Forward Neural Network (FFNN). The output from the FFNN generates the pixel classification. From the pixellevel segmented image, region-level classification is achieved with 4-connected labelled pixels and form regions. Following the region classification, area classification and image level classification are applied with region growing operation to obtain an overall size of area i.e., drusen. All these three classification steps are based on a rule-based approach which utilizes minimum and maximum border intensity of the region, average gradient and size of the region etc. The algorithm was evaluated on a set of 119 images, 62 of which contained drusen, 38 ofwhich were of healthy retinas, and 19 of which contained non-drusen abnormalities. Overall, the method achieved $87 \%$ percent accuracy for drusen and non drusen detection. The method also classified images as normal, large few drusen, large many drusen, fine few drusen, fine many drusen and abnormal drusen. The method achieved $71 \%$ accuracy for classification of images. The method did not consider any soft and hard drusen, and distinct and indistinct drusen classification which is an essential task for early AMD detection and AMD grading.

Kose et al. [34] proposed a method for AMD detection based on identification of healthy and degenerative regions in the retinal. They used the statistical properties of healthy textures and used thresholding to segment the region. The method considers a reference characteristic image texture and compares the texture of the sample image (which is to be graded for AMD) to a small to larger region and if it is within a certain threshold, the area is map as normal. The blood vessel edges are computed using Sobel operation and parallel edges are obtained as vessel edges. Based on the density of the vessel edges the method approximates the area with maximum density values as optic disc. Following this, the inverse segmentation is implemented to obtain the area of degenerations and compute the total area of degeneration. 30 images were included for testing the algorithm. The method achieved $84.06 \%$ - 92.64\% accuracy on small degeneration detection, $89.89 \%$ 96.06\% medium degeneration detection and $90.67 \%-95.16 \%$ large degeneration detection in the fundus images. The method applies 
a simple method for degeneration detection, however, it should be suffered from image qualities and variation of the image illumination and contrast as simple matching is applying between the textures of the images. Besides this, the method should be confused with drusen and exudates, which are pathologies for MD and retinopathy. Also, the method did not report anything about distinct and indistinct soft drusen a key for AMD stage identification.

Parvathi and Devi [28] developed a method for drusen detection which is based on a bank of Log-Gabor filters and topographic models. The method uses retinal green channel image and applies a bank of Gabor filters with different scale and orientations to capture the high frequency components (i.e., drusen edges). As the optic disc and blood vessels also have similar properties, a preprocessing step is applied to remove these objects from the image. The Gabor filters responses return maximum energy at the locations of drusen. Thus, projecting the $2 \mathrm{D}$ image function as a surface in $3 \mathrm{D}$ space leads to a different perspective of drusen as they have hilly profiles, which is measured by surface curvature. The major drawback of the technique is in the detection of soft drusen which have less edge effect or faint boundary.

Barriga et al. [29] proposed as method for lesion phenotyping on AMD which focused on developing an automated AMD grading system. Amplitude modulation - frequency modulation (AM-FM) is used to generate multi-scale features for classifying pathological structures, such as vessels, retinal background, hard and soft drusen. AM-FM methods are applied at different scales for representing an input image as a sum of AM-FM components. In the components, the AM functions characterize slowly-varying image intensity variations; whereas the FM functions characterize fast-varying image intensity variations. Eleven different combination of scales $(\mathrm{CoS})$ are used for extracting the dominant AM-FM estimated from different frequency ranges. For each $\mathrm{CoS}$, the histograms of the instantaneous amplitude, instantaneous frequency and angle of instantaneous frequency are used to create a feature vector for encoding the characteristics related with the structure presented in the region of interest (ROI). The CoS method is applied on three colour spaces: RGB, HSV and the OHTA. Following this, Partial Least Squares is used to classify the features for the ROIs. The method considered 120 images for accuracy testing and achieved $100 \%$ sensitivity and specificity for categorizing different retinal structures such as blood vessel, background and soft drusen. However, distinction between hard and soft drusen is achieved $96 \%$ accuracy. The classification of distinct and indistinct drusen is not performed that is one of the most important criteria for AMD grading.

Agurto et al. [32] described and evaluated the performance of the AM-FM algorithm [29]. 2247 retinal photographs were obtained of 822 patients with three-field of view. Ground truth was provided for the presence of pathologic conditions, including drusen, micro aneurysms, hemorrhages, exudates and others. The algorithm [29] was used to report on the presence and absence of AMD and diabetic retinopathy. The system achieved an average area under the ROC curve (AUC) of 0.77 on Rist dataset and 0.73 on UTHSCSA dataset for drusen detection.

Lee et al. [27] proposed a method for disease and non-disease retina which is based on texture information and clustering for selection of drusen and non drusen images. Multiscale top-hat filter is used along with other non-homogeneous texture descriptor such as multi-channel wavelet representation and construct a feature space to cluster the texture based on similarity.The clustering starts with a low frequency texture subspace to assign similarity assignments among classes and then gradually refine membership assignments by incorporating higher frequency subspaces during clustering. Once a representation is found for structural texture cues, an unsupervised learning method is applied to separate the feature space into non overlapping clusters to learn a partition function. Following this, a partition function is used to assign the cluster membership for a new test sample by optimizing for the closest similarity match with highest probability. The method is tested with four sample images with different types of drusen texture. The accuracy varies between $71.7 \%$ and $92.2 \%$. The method is tested with a small sample size and also does not show any result for drusen type identification a vital task for AMD grading.

Freund et al. [33] developed a method for AMD and non AMD image classification based on multiscale analysis and Support Vector Data Description (SVDD). At first, intensity based equalization algorithm is applied in the preprocessing step. Following this, Mexican hat wavelet is used to derive a set of feature values (the products of $41 \mathrm{D}$ convolutions on 10 wavelet dilations) for each pixel in the image. Then SVDD algorithm is implemented using Gaussian radial basis function (RBF) to classify the pixels into a binary image. Finally, the blood vessels detected by Canny edge detector and morphological erosion and dilation are removed. The method considers an image as AMD based on the drusen presence which is incorrect.

Priya and Aruna [41] proposed a method for dry and wet AMD detection using Discrete Wavelet Transform (DWT) and Probabilistic Neural Network (PNN). DWT is used to obtain the coefficients in horizontal, vertical and diagonal directions. Kirsch template is applied to obtain the edge information including direction. Optic disc information is obtained through vessel density and thickness measurement. Then PNN is applied to classify the dry and wet AMD. The method achieved 96\% accuracy in dry and wet AMD classification. A large variation of drusen types with image variation can show the robustness of the technique.

\section{Thresholding based segmentation}

Soliz et al. [30] developed a quantitative assessment method for maculopathy using colour retinal images. They used Gaussian smoothing and probability based thresholding method to quantify drusen and assess longitudinal changes in retinal images presenting with maculopathy following Wisconsin Age-related Maculopathy Degeneration Grading Scheme. Initially, colour images were produced from colour slides. Blue colour channel was used to produce optic disc and illumination information through enhancing the regions. Then Median filtering (size of $3 \times 3$ ) and following this Gaussian smoothing with a large sigma of 50 are applied on green channel to remove impulse noise and smooth the image respectively. A subtracted image between the original green channel and the smoothed image is obtained. Then the image is split into 27 regions and produces the histogram for each of them. Following this, each region is converted into a binary image by considering a percentage of the high intensity pixels in the histogram belong drusen regions. Finally the region growing technique is applied to map the regions and quantify their areas. A series of manual control tools are provided for a grader (or rater) to further improve the segmentation results. The method achieved up to $71 \%$ accuracy to quantify drusen in an image. The longitudinal changes were also computed and analyzed by the output images. However, the agreement between the graders on different drusen sizes was only $67 \%$.

Smith et al. proposed a method [43] for morphometric analysis of drusen based on macular background reflectance thresholding. Initially non-uniform illumination due to retinal reflectance is corrected by 
a common shading correction method on each channel of RGB. The background pattern in the green channel is leveled by an algorithm based on the elliptically concentric geometry of the reflectance in the normal macula. A semiautomatic method including luteal pigment correction step and interactive background leveling step is applied to obtain the gray scale values of all structures. This method is performed within defined elliptical boundaries which are raised sequentially until a uniform background is obtained. The segmentation of drusen and area measurements in the central and middle subfields $(1000 \mu \mathrm{m}$ and 3000 $\mu \mathrm{m})$ is performed by uniform thresholds. The semi-automated method showed good reproducibility of the drusen segmented area. The area measurement disagreement between the manual grading and semiautomatic method were $2.8+/-3.4 \%$ in the central subfield and $2.2+/$ $2.7 \%$ in the middle subfield. However, the validation was performed with a small sample size (12 images) and also intra-grader distance is not computed to show the robustness of the system. Recently, the method was validated with 2 large independently population based data set Netherlands Genetic Isolate Study and Columbia Macular Genetics Study [35]. In the validation method, 358 colour slides from the first study were used for manual grading and their digital images were obtained for grading by using the semiautomatic software. A weighted Cohen's kappa, $\mathrm{K}$ statistics showed the weighted $\mathrm{k}$ values were 0.61 , 0.62 and 0.76 for small, intermediate and large drusen, respectively. 164 images from the second study were used for the validation. The corresponding weighted $\mathrm{K}$ values were $0.86,0.84$ and 0.80 for the centre, inner and outer circles of macular region, respectively.

Later Smith et al. developed an automated method [36] based on [43]. In the previous semi-automated method, an operator is needed to select the background area in the retinal image and adapt the threshold value of the pixel intensities. In the automated method, the initial background is selected by Otsu method which is applied by dividing the image into two zones: the central (circle with diameter of 1000 $\mu \mathrm{m}$ ) and middle (annulus between the diameters of $1000 \mu \mathrm{m}$ and 3000 $\mu \mathrm{m})$ subfields. Then sequential automated background leveling and histogram-based thresholding are applied to segment the drusen areas. The segmented areas are then compared on a pixel-by-pixel basis to the areas from manual drawings. The method achieved sensitivity of 0.42 to 0.86 (median 0.70 ) and specificity from 0.53 to 0.98 (median 0.81 ). In drusen area measurement the method produces the difference with ground truth images ranged from $-6.7 \%$ to $12.7 \%$. The method did not produce the indentified drusen subtype values and AMD stage from the retinal images.

Rapantzikos and Zervakis [7,31] proposed a method for drusen segmentation which applies histogram-based adaptive local thresholding method on illumination corrected and contrast enhanced images. A homomorphic filtering is used for non-uniform illumination compensation and a multilevel variant of histogram equalization is developed for image contrast enhancement. Global threshold is applied for testing general symmetry or asymmetry. Two quotients, namely the differences |mean-median| and |mean-mode|, are used for the measurement of symmetry/asymmetry in a gray-scale distribution under a considered region. If these two quotients are small, the grayscale distribution under consideration is classified as symmetric, otherwise asymmetric. Then histogram adaptive thresholding is applied at local regions of the image depending on various shape properties (e.g., skewness and kurtosis, etc.) of the corresponding histogram. The adaptive selection of the threshold follows two roles: kurtosis decision role while the histogram is totally or almost symmetric and skewness decision role while the histogram is totally or almost asymmetric.
The results showed that the method achieved up to $100 \%$ accuracy in drusen pixel detection. However, it is not clear that if the method can perform with similar accuracy for soft drusen detection.

Liang et al. [37] developed a method for drusen detection which is based on macular region mapping, vessel removal and intensity thresholding. At first edge detection algorithm is applied on red and green colour channel and used their summation to produce the binary edges based on thresholding. Morphological dilation is applied to smooth the boundaries of the detected regions and produce the mask to remove the vessel regions. The macula area is mapped based on optic disc intensity and macula intensity (i.e., dark pixel) and their positional information. Largest group of dark pixels are consider as the macula area and circular grid is computed based on the macula centre. The drusen from the outermost circular region are detected based on intensity thresholding from green channel image. The method reported sensitivity and specificity of 0.75 in drusen identification.

Bhuiyan et al. [44] proposed a method for detecting drusen and quantifying drusen size in macular region from standard colour retinal images for early diagnosis of Age related Macular Degeneration (AMD). Colour retinal images were used which were captured by Canon D60 non-mydriatic camera for genetic and epidemiology study. Local intensity distribution, adaptive intensity thresholding and edge information were used to detect potential drusen areas. For validation, 50 images were considered with various types of drusen. The proposed method detected the presence of any drusen with $100 \%$ accuracy $(50 / 50$ images). For the drusen area segmentation accuracy(DAA), 12 images were selected, and an expert grader marked the drusen regions in pixel level. The quantified areas were used to compute the sensitivity and specificity by comparing the drusen detected output images with the hand-labelled ground truth (GT) images. Considering drusen pixel positive and background pixel negative, we used the following formula and computed the sensitivity and specificity: Sensitivity $=\mathrm{TP} /(\mathrm{TP}+\mathrm{FN})$ and Specificity $=\mathrm{TN} /(\mathrm{TN}+\mathrm{FP})$, where, $\mathrm{TP}$ is the number of overlapped positive pixels in drusen and GT image FP is the number of positive pixels in drusen image does not match with GT image, TN is the number of overlapped negative pixels in drusen and GT image, FN is the number of negative pixels in drusen image does not match with GT image. For drusen detection accuracy (pixel level), mean sensitivity and specificity values of $74.94 \%$ and $81.17 \%$, respectively. For drusen subtypes we achieved $79.59 \%$ accuracy in intermediate drusen and $82.14 \%$ in soft drusen which is a highly significant result for early and intermediate AMD detection.

Barkat and Madjarov [38] investigated a semiautomatic method on drusen quantification for clinical trials. An operator is needed to define a region of interest using a user interface to allow the system to select the drusen region robustly.Variation of the brightness level is adjusted for increasing the efficiency of drusen detection. Then an intensity index is defined based on several cycles of drusen detection to optimize the algorithm's performance. The drusen detection result obtained by this algorithm was compared with manually measured drusen areas that showed $86 \%$ sensitivity and $93 \%$ specificity. The method did not consider drusen types to measure the AMD severity.

Morgan et al. [45] proposed a method for extraction and quantification of macular drusen using fundus photograph. In the automated quantification phase, the method first applies median filtering to reduce impulse noise. Then it subdivides the image into several small regions and computed the mean pixel intensity value. The threshold value is computed with considering the adjacent regions 
and pixel location to obtain the brighter pixel location. Macular region is selected manually from the digital image and the Polaroid prints to quantify the drusen areas. The method compared with a manual method which produced the quantified drusen area from Polaroid prints. The results showed that a significant number of drusen area was miss calculated with up to $100 \%$ difference between manual and automatic method which is a major drawback of the system.

\section{Clustering}

Quellec et al. [46] proposed a method for the detection of drusen and other lesions (e.g., retinopathy) based on a feature space which is automatically derived from a set of reference image samples representing target lesions. The reference image samples are obtained either from an expert or a data driven approach. Factor analysis is used to derive the filters generating this feature space from reference samples. Then the new image is classified using this feature space. The approach is tested on the detection of AMD, by training it to differentiate drusen from Stargardt's disease lesions and achieved an area under the receiveroperating characteristic curve (AUC) $=0.85$ on a set of 300 manually detected drusen from 15 images. The method is very fast (less than a second for a standard PC); however, it did not consider the validation for soft drusen detection or other drusen subtypes detection accuracy.

Hijazi et al. [47] developed a method which is based on spatialhistogram and Case Base Reasoning approach for classifying image into AMD and non-AMD. An image firstly goes through a series of preprocessing, as image enhancement, object segmentation and noise reduction. Then the image is portioned into a number of regions and each region is represented in a spatial histogram that stores colour and spatial image information. A class separability measure is applied for selecting the most appropriate spatial-histograms from a set of processed images. These selected histograms, each representing one image, are stored in the form of time series curves as Case Base. A new case will go the same preprocessing and histogram generation steps. Then the case is classified by finding the most similar case (curve) in the Case Base. Similarity checking is achieved using the dynamic time warping (DTW) time series analysis technique. The method achieved overall 77\% accuracy in AMD and non-AMD image classification. The major drawback of the paper is it simplifies AMD as the presence of any object such as drusen which is trivial AMD.

\section{Edge and template matching}

Mora et al. [39] proposed a method for automatic drusen detection and quantification using retinal images. First, an iterative cubic smoothing spline fitting method is applied for uneven illumination correction. Then a Root Mean Square contrast calculation is applied for image contrast normalization. Following this, gradient based segmentation algorithm is used to isolate drusen and Modified Gaussian function is fitted to decide the region as drusen or noise. The validation of the methodology was performed on twenty-two retinal images. A significant variability was shown within the experts' grading (on average $43 \%$ ) on total affected areas. The accuracy on a pixel-topixel comparison, the method achieved an average sensitivity of 0.68 and specificity of 0.96 .

Remeseiro et al. [40] developed a method for automatic drusen detection from digital images. The method adopts template matching and threshold operation for drusen region selection. Two Gaussian templates are utilized to produce cross-correlation with the image region. A threshold is selected based on the empirical value and a seed point is selected to apply seeded region growing operation. The region growing process finishes when no more pixels can be added to any existing region or if the region exceeds the maximum size of 150 pixels. The method was validated with four images which included 11 drusen and later 16 drusen were inserted artificially (due to lack of images). In drusen region identification the method achieved $83 \%$ sensitivity and $87 \%$ specificity.

GA detection: Our research did not find any study for automated GA detection using colour imaging. Fluorescence angiography [48] is the standard process for automated quantification of GA. A semiautomated GA grading system is reported which also includes drusen grading - as follows.

Grading systems: Scholl et al. [49] proposed a semi-automated method for grading lesions responsible to measure AMD severity which is based on colour fundus imaging. Age related maculopathy (ARM) and AMD were graded using a grid to define macular subfields and standard circles to define the size of the lesions. Inter-observer variability was assessed by having three retinal specialists evaluate the colour slides and intra-observer variability by re-grading the same set. The interobserver agreement for hard drusen was $70-89 \%$ (kappa $=0.26-0.63$ ) and intermediate soft drusen $76-94 \%$ (kappa $=0.27-0.69$ ). Agreement ranged between $87 \%$ and $100 \%$, for larger drusen.

Overall, the best performance methods can be summarized as follows. We note that the methods reported accuracies are not on the same dataset and therefore, not comparable to each other. We only list the approaches with highest accuracy.

- Drusen absent and present - reported accuracy is $100 \%$ by most of the methods.

- Drusen pixel classification - overall accuracy $87 \%$ and sensitivity is $86 \%$ and specificity is $93 \%$ [38].

- Drusen size or region - over all accuracy is 96\% [29].

- Dry and wet AMD identification - overall 96\% [41].

In summary, a large number of researches have been reported for drusen detection and quantification. However, most of the techniques focused on drusen absent or present, or drusen pixel identification. No method has reported the drusen detection accuracy with the presence of hyper-pigmentation. Another limitation of the current studies is the lack of ability to classify hard and soft drusen, and soft distinct and indistinct drusen. However, it is essential information for automated AMD severity grading and is still an open area of study. An AMD grading framework can be incorporating modules such as blood vessel, optic disc and macula area mapping, drusen detection and classification, quantification and total drusen area in the macula for severity measurement or grading. This system is shown in Figure 7.

\section{Telemedicine for AMD Screening}

Telemedicine is the use of telecommunication and information technologies in order to provide clinical health care at a distance that helps eliminate distance barriers and can improve access to medical services that would often not be consistently available in distant rural communities. Because ophthalmology is largely dependent on visual information, it is an ideal specialty for telemedicine. The contrast with live video-conferencing, which is currently limited by electronic transmission rates and specialist online availability, "tele-ophthalmology" could be utilized between primary health care 


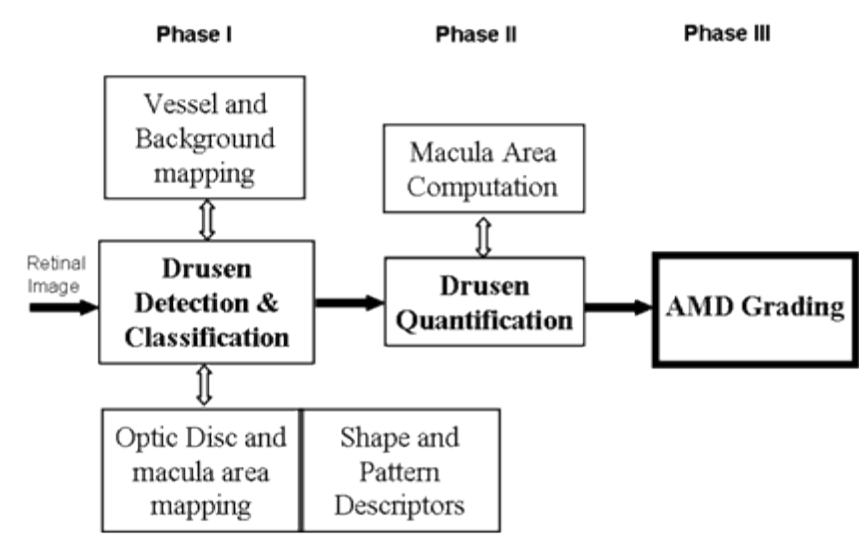

Figure 7: A framework for AMD grading.

practitioners, optometrists and ophthalmic specialists, or between different ophthalmic units [50]. Telemedicine services reduce healthcare costs and enables early detection of blinding eye conditions, e.g., diabetic retinopathy and AMD [51]. An example, telemedicine has been used to aid prison medical officers in diagnosing ophthalmic complaints, and thus reducing costs and potential complications of prisoners attending specialist medical centres [52]

Several feasibility studies demonstrate the enormous usefulness of the technology which includes the communities living in rural and remote areas [53-57]. However, our recent literature search found only a few telemedicine screening program $[58,59]$; both showed very promising results on AMD screening through telemedicine. Pirbhai and Sheidow [58] showed that telemedicine can be used to diagnose AMD patient with high confidence using colour fundus photograph. The automatic system could achieve $89.2 \%$ sensitivity and $99.1 \%$ specificity on detecting choroidal neovascularization. For the presence of RPE geographic atrophy the specificities are up to $86.8 \%$. Very few treatable lesions are missed in AMD patients. The other telemedicine study for AMD screening mainly reported the efficiency of AMD diagnosis program [59]. The Canadian project demonstrates that unnecessary consultations can be avoided through telemedicine based screening program.

Telemedicine have been proven to be highly effective for cost saving and an efficient healthcare system. Bashshur et al. [60] emphasized the potentiality of telemedicine platform in healthcare system which models united states healthcare system, i.e., a developed country. This technology will be more effective for under developed countries which have a large population burden and less specialists doctors [61]. Current internet infrastructure (for telemedicine [51] in any country should provide this services. Another benefit of the telemedicine platform is that it can facilitate mass screening of AMD from urban and rural population based on small community clinics and optometry shops. Therefore, using telemedicine should be able to identify patients at their early stage of AMD more effectively and can prevent late AMD which will impact hugely on the socio-economic status of a country.

\section{Future Research Direction}

Future research of this imaging modality should focus on robust software and using multisensory imaging capabilities to capture AMD pathologies with confidence. In our discussion we mentioned that we have not received a single paper on soft drusen classification which can be a key measurement for automatic AMD severity identification.
Although, there has been a considerable work on drusen detection, none of them addresses drusen classification such as hard and soft drusen and distinct and indict drusen which are the key measure of severity of drusen and a key for automated early AMD screening. The most challenging issues on drusen classification are the varieties of size, shape and intensity distribution for the same type of drusen. In addition, it is often difficult to find the boundaries of drusen and can also have a similar appearance to other lesions, such as cotton wool spots. Some faint drusen can also appear similar to normal features of the retina, such as the background pattern caused by the choroidal vessels [34].

Future research for AMD screening should focus on automated retinal image analysis using multimodal imaging and using telemedicine platform for mass screening of AMD from rural and remote areas. As there is no treatment to reverse the AMD, therefore using telemedicine platform will be highly effective for detecting AMD at early stage and start treatment to prevent further degeneration. To achieve such a system we need fully automated AMD screening method. Therefore, we need a highly accurate method for AMD pathology classification, fusion of data from multiple imaging modalities and using the information as complement to each other. An integrated system with telemedicine platform and automated image analysis from multimodal imaging is the future generation decision support for identification of AMD severity.

\section{Conclusion}

In this paper, we examined the existing techniques for automated screening of AMD using colour fundus imaging. We emphasized that to date; there is no drug or treatment to cure AMD. Therefore, the only feasible option is to prevent the incidence of AMD and avoid this fatal eye disease as well as unnecessary vision loss. For this, a mass screening system that enables remote diagnosis of AMD should be implemented for taking preventative measures in the early stage of AMD. We discussed and reviewed the automated methods for detecting AMD pathologies such as drusen and geographic atrophy (GA). We intended to provide a brief description, highlighting the key points of the performance measurement and a framework for the existing research. We outlined the open problems for automated AMD identification systems using colour fundus imaging with future research directions.

\section{References}

1. Bartlett $H$, Eperjesi $F$ (2007) Use of fundus imaging in quantification of agerelated macular change. Surv Ophthalmol 52: 655-671.

2. Wong TY, Liew G, Mitchell $P$ (2007) Clinical update: new treatments for agerelated macular degeneration. Lancet 370: 204-206.

3. Van Newkirk MR, Nanjan MB, Wang JJ, Mitchell P, Taylor HR, et al. (2000) The prevalence of age-related maculopathy: the visual impairment project. Ophthalmology 107: 1593-1600.

4. Wong TY, Rogers SL (2007) Statins and age-related macular degeneration time for a randomized controlled trial? Am J Ophthalmol 144: 117-119.

5. Wong TY, Chakravarthy U, Klein R, Mitchell P, Zlateva G, et al. (2008) The natural history and prognosis of neovascular age-related macular degeneration: a systematic review of the literature and meta-analysis. Ophthalmology 115 116-126.

6. Covert D, Berdeaux G, Mitchell J, Bradley C, Barnes R (2007) Quality of life and health economic assessments of age-related macular degeneration. Surv Ophthalmol 52 Suppl 1: S20-25.

7. Rapantzikos K, Zervakis M, Balas K (2003) Detection and segmentation of drusen deposits on human retina: potential in the diagnosis of age-related macular degeneration. Med Image Anal 7: 95-108. 
Citation: Bhuiyan A, Xiao D, Yogesan K (2014) A Review of Disease Grading and Remote Diagnosis for Sight Threatening Eye Condition: Age Related Macular Degeneration. J Comput Sci Syst Biol 7: 062-071. doi:10.4172/jcsb.1000139

8. Klein R, Davis MD, Magli YL, Segal P, Klein BE et al (1991) The Wisconsin age-related maculopathy grading system. Ophthalmology 98: 1128-1134.

9. Gregor Z, Bird AC, Chisholm IH (1977) Seniledisciform macular degeneration in the second eye. Br J Ophthalmol 61: 141-147.

10. Smiddy WE, Fine SL (1984) Prognosis of patients with bilateral macular drusen Ophthalmology 91: 271-277.

11. Strahlman ER, Fine SL, Hillis A (1983) The second eye of patients with senile macular degeneration. Arch Ophthalmol 101: 1191-1193.

12. Niemeijer M, van Ginneken B, Russell SR, Suttorp-Schulten MS, Abràmoff MD (2007) Automated detection and differentiation of drusen, exudates, and cotton-wool spots in digital color fundus photographs for diabetic retinopathy diagnosis. Invest Ophthalmol Vis Sci 48: 2260-2267.

13. Freund KB, Yannuzzi LA, Rosenthal DR (2008) Age-Related Macular Degeneration. The Macula Foundation, Inc.

14. de Jong PT (2006) Age-related macular degeneration. N Engl J Med 355: 1474-1485.

15. Bressler NM, Bressler SB, Fine SL (1988) Age-related macular degeneration. SurvOphthalmol 32: 375-413.

16. Jager RD, Mieler WF, Miller JW (2008) Age-related macular degeneration. N Engl J Med 358: 2606-2617.

17. Baumann B, Gotzinger E, Pircher M, Sattmann H, Schuutze C, et al. (2010) Segmentation and quantification of retinal lesions in age-related macular degeneration using polarization-sensitive optical coherence tomography. J Biomed Opt15:1-21.

18. Zhang N, Hoffmeyer GC, Young ES, Burns RE, Winter KP, et al. (2007) Optical coherence tomography reader agreement in neovascular age-related macular degeneration. Am J Ophthalmol 144: 37-44.

19. Nazemi PP, Fink W, Lim JI, Sadun AA (2005) Scotomas of age-related macular degeneration detected and characterized by means of a novel threedimensional computer-automated visual field test. Retina 25: 446-453.

20. Snellen Chart Wikipedia

21. Fleckenstein M, Schmitz VS, Holz FG (2010) Fundus Autofluorescence Imaging in Clinical Use. In: Review of Ophthalmology

22. Schardosim L, Scharcanski J, Santos Dd, Stuchi Je (2011) Motion Detection in Infrared Retinal Image Sequences. IEEE Conference on Instrumentation and Measurement Technology.

23. Pemp B, Schmetterer $L$ (2008) Ocular blood flow in diabetes and age-related macular degeneration. Can J Ophthalmol 43: 295-301.

24. Kanagasingam Y1, Bhuiyan A2, Abràmoff MD3, Smith RT4, Goldschmidt L5, et al. (2014) Progress on retinal image analysis for age related macular degeneration. ProgRetin Eye Res 38: 20-42.

25. Bird AC, Bressler NM, Bressler SB, Chisholm IH, Coscas G, et al. (1995) An international classification and grading system for age-related maculopathy and age-related macular degeneration. The International ARM Epidemiological Study Group. SurvOphthalmol 39: 367-374.

26. Brandon L, Hoover A (2003) Drusen Detection in a Retinal Image Using MultiLevel Analysis. LNCS: 618-625.

27. Lee N, Laine AF, Smith TR (2008) Learning non-homogenous textures and the unlearning problem with application to drusen detection in retinal images. Academic Commons: 1215-1218.

28. Parvathi SS, Devi N (2007) Automatic Drusen Detection from Colour Retina Images. In: International Conference on Conference on Computational Intelligence and Multimedia Applications: 377-381.

29. Barriga ES, Murray V, Agurto C, Pattichis MS, Russell S, et al.(2009) Multiscale AM-FM for lesion phenotyping on age-related macular degeneration. INSPEC: $1-5$.

30. Soliz P, Wilson M, Nemeth S, Nguyen P (2002) Computer-aided methods for quantitative assessment of longitudinal changes in retinal images presenting with maculopathy. SPIE 4681:159-170.

31. Rapantzikos K, Zervakis M (2002) Nonlinear enhancement and segmentation algorithm for the detection of age-related macular degeneration (AMD) in human eye's retina. In: Proceedings of International Conference on Image Processing. Greece: 1055 - 1058.
32. Agurto C, Barriga ES, Murray V, Nemeth S, Crammer R, et al. (2011) Automatic detection of diabetic retinopathy and age-related macular degeneration in digital fundus images. Invest Ophthalmol Vis Sci 52: 5862-5871.

33. Freund DE, Bressler N, Burlina P (2009) Automated detection of drusen in the macula. IEEE International Symposium on Biomedical Imaging: From Nano to Macro: 61-64.

34. Köse C, Sevik U, Gençalioğlu O, Ikibaş C, Kayikiçioğlu T (2010) A statistical segmentation method for measuring age-related macular degeneration in retinal fundus images. J Med Syst 34: 1-13.

35. Smith RT, Sohrab MA, Pumariega NM, Mathur K, Haans R, et al. (2011) Drusen analysis in a human-machine synergistic framework. Arch Ophthalmol 129: 40-47.

36. Smith RT, Chan JK, Nagasaki T, Ahmad UF, Barbazetto I, et al. (2005) Automated detection of macular drusen using geometric background leveling and threshold selection. Arch Ophthalmol 123: 200-206.

37. Liang Z, Wong DW, Liu J, Chan KL, Wong TY (2010) Towards automatic detection of age-related macular degeneration in retinal fundus images. ConfProc IEEE Eng Med BiolSoc 2010: 4100-4103.

38. Barakat MR, Madjarov B (2004) Automated drusenquantitaion for clinical trials Invest Ophthalmol Vis Sci.

39. Mora AD, Vieira PM, Manivannan A, Fonseca JM (2011) Automated drusen detection in retinal images using analytical modelling algorithms. Biomed Eng Online 10: 59

40. Remeseiro B, Barreira N, Calvo D, Ortega M, Penedo MG (2009) Automatic Drusen Detection from Digital Retinal Images. Springer-Verlag Berlin, Heidelberg, pp: 187-194.

41. Priya R, Aruna P (2011) Automated diagnosis of Age-related macular degeneration from color retinal fundus images. IEEE: 227-230.

42. Wu D, Zhang M, Liu JC, Bauman W (2006) On the adaptive detection of blood vessels in retinal images. IEEE Trans Biomed Eng 53: 341-343.

43. Smith RT, Nagasaki T, Sparrow JR, Barbazetto I, Klaver CC, et al. (2003) A method of drusen measurement based on the geometry of fundus reflectance. Biomed Eng Online 2: 10.

44. Bhuiyan A, Kawasaki R, Sasaki M, Lamoureux E, Ramamohanarao K, et al (2013)Drusen Detection and Quantification for Early identification of age related macular degeneration using color fundus imaging. J ClinExpOphthalmol4:305.

45. Morgan WH, Cooper RL, Constable IJ, Eikelboom RH (1994) Automated extraction and quantification of macular drusen from fundal photographs. Aust N Z J Ophthalmol 22: 7-12

46. Quellec G, Russell SR, Abramoff MD (2011) Optimal filter framework for automated, instantaneous detection of lesions in retinal images. IEEE Trans Med Imaging 30: 523-533.

47. Hanafi M, Hijazi A, Coenen F, Zheng Y (2010) Retinal Image Classification for the Screening of Age-related Macular Degeneration. In: SGAI International Conference on Artificial Intelligence: 325-338.

48. Lee N, Laine AF, Smith RT (2007) A hybrid segmentation approach for geographic atrophy in fundus auto-fluorescence images for diagnosis of agerelated macular degeneration. Conf Proc IEEE Eng Med BiolSoc 2007: 49654968.

49. Scholl HP, Peto T, Dandekar S, Bunce C, Xing W, et al. (2003) Inter- and intraobserver variability in grading lesions of age-related maculopathy and macular degeneration. Graefes Arch ClinExpOphthalmol 241: 39-47.

50. Patton N, Aslam TM, MacGillivray T, Deary IJ, Dhillon B, et al. (2006) Retinal image analysis: concepts, applications and potential. Prog Retin Eye Res 25 99-127.

51. Yogesan K, Reinholz F, Constable IJ (2010) Tele-Diabetic Retinopathy Screening and Image-Based Clinical Decision Support: Automated Image Detection of Retinal Pathology. CRC Press, New York, USA

52. Yogesan K, Henderson C, Barry CJ, Constable IJ (2001) Online eye care in prisons in Western Australia. J TelemedTelecare 7 Suppl 2: 63-64.

53. Abramoff MD, Suttorp-Schulten MS (2005) Web-based screening for diabetic retinopathy in a primary care population: the EyeCheck project. Telemed J E Health 11: 668-674. 
Citation: Bhuiyan A, Xiao D, Yogesan K (2014) A Review of Disease Grading and Remote Diagnosis for Sight Threatening Eye Condition: Age Related Macular Degeneration. J Comput Sci Syst Biol 7: 062-071. doi:10.4172/jcsb.1000139

54. Joshi GD, Sivaswamy J (2011) DrishtiCare: a telescreening platform for diabetic retinopathy powered with fundus image analysis. J Diabetes SciTechnol 5: 2331.

55. Lorenz B, Spasovska K, Elflein H, Schneider N (2009) Wide-field digital imaging based telemedicine for screening for acute retinopathy of prematurity (ROP). Six-year results of a multicentre field study. Graefes Arch Clin Exp Ophthalmol 247: $1251-1262$

56. Liesenfeld B, Kohner E, Piehlmeier W, Kluthe S, Aldington S, et al. (2000) Atelemedical approach to the screening of diabetic retinopathy: digital fundus photography. Diabetes Care 23: 345-348.

57. Murakami Y, Silva R, Jain A (2009) Stanford Univesrity Network for Diagnosis of Retinopathy of Prematurity (SUNDROP): 12-month experience with telemedicine screening. $\mathrm{Br} J$ Ophthalmol9:1456-1460.

58. Pirbhai A, Sheidow T, Hooper P (2005) Prospective evaluation of digital nonstereo color fundus photography as a screening tool in age-related macular degeneration. Am J Ophthalmol 139: 455-461.

59. Gagnon L (2007) Telemedicine offers effective screening option for AMD Ocular Surg News 25:18.

60. Bashshur RL, Shannon GW, Krupinski EA, Grigsby J, Kvedar JC, et al. (2009) National telemedicine initiatives: essential to healthcare reform. Telemed $\mathrm{J} \mathrm{E}$ Health 15: 600-610.

61. Eccles N (2012) Telemedicine in Developing Countries: Challenges and Successes. Harvard College Periodical Review (HCGHR). 